a completely successful mitral valvotomy, or repair of an atrial septal defect, it is probably no longer needed. The effectiveness of quinidine, after conversion with direct-current shock, to lessen the relapse rate is being further studied.

No complication in the present series has been serious. Extreme care is required and proper insulation of the apparatus is essential, especially when used at the time of thoracotomy. Ventricular tachycardia and fibrillation after direct-current shock in dogs appear to be related to the height of the voltage and the amount of energy used (Peleška, 1963). Our present plan for version is to use no more than four shocks, with energy increasing from 150 to 250,350 , and 400 watt sec.

Relapse after successful direct-current shock seems most frequent if the left atrial pressure and volume remain above normal once sinus rhythm has been restored. This may occur in mitral-valve disease and in some of the cardiomyopathies. In the patients with "lone" atrial fibrillation there has been an as yet unexplained high relapse rate. Many of the patients with cardiomyopathy have been alcoholic, and failure to remove the provocative agent may contribute to their relapse. The need to repeat the procedure frequently should not arise; if the rhythm is constantly unstable, well-controlled atrial fibrillation is preferable to repeated changes of rhythm.

In conclusion, a new method of treating arrhythmias has proved better than old methods for many patients, and it should have an established place for future practice.

\section{Summary}

Fifty patients who had atrial fibrillation, atrial flutter, or atrial tachycardia, resistant to treatment with drugs, were treated with direct-current shock. In 43 of these sinus rhythm was successfully achieved. The method is described and indications and contraindications for this form of treatment are given.

Complications, which were few, are described. The results of a follow-up extending to nine months after successful treatment are given.

We wish to thank the physicians and surgeons of the National Heart Hospital, and Drs. William Barratt, Richard Bomford, and Walter Somerville, who referred patients; Drs. John Beard and Alan Gilston, who gave the anaesthetics; Sister Curry, theatre superintendent of the National Heart Hospital, and her staff for willing assistance; and $\mathrm{Mr}$. John Norman, chief technician, the Institute of Cardiology, and his staff for technical help.

ADDENDUM.-Since this paper was written a further 59 patients with arrhythmias have been treated with direct-current shock. The results in them have been similar and our conclusions the same.

\section{REFERENCES}

Berman, R., Sadaff, C. M., and Gordon, G. B. (1953). Minn. Med. 36, 1052 .

Hecht, H. H., Osher, W. J., and Samuels, A. J. (1951). f. clin. Invest., 30, 647 .

Lown, B., Amarasingham, R., and Neuman, J. (1962a). F. Amer. med. Ass., 182, 548 .

Neuman, J., Amarasingham, R., and Berkovits, B. V. (1962b). Amer. F. Cardiol., 10, 223.

McDonald, L., Resnekov, L., and Ross, D. (1963). Lancet, 2, 708.

O'Brien, K., Resnekov, L., and McDonald, L. (1964). Brit. Heart f., 26 $1,137$.

Oram, S., Davies, J. P. H., Weinbren, I., Taggart, P., and Kitchen, L. D. (1963). Lancet, 2, 159.

Peleška, B. (1963). Circulat. Res., 13, 21.

\title{
Investigation of Failure of Artificial Pacing
}

\author{
EDGAR SOWTON,*† M.D., M.R.C.P. ; J. G. DAVIES*
}

The use of artificial pacemakers in the treatment of StokesAdams disease has increased rapidly over the past few years, and many patients who were previously bedridden or suffered from recurrent syncopal attacks have been enabled to live relatively normal lives. The combination of electronic and clinical attention required by patients with artificial pacemakers can best be provided by the specialized centre where the pacemaker was originally inserted, but as the number of patients increases, the problems associated with this form of therapy are likely to be encountered in the future in many hospitals where the staff have little experience of the complications which may arise. This paper is intended to provide a guide for the initial investigation of a patient presenting with a history suggesting failure of pacing and is based on information obtained from a series of over 80 patients paced for periods of up to four years. It does not assume a knowledge of electronics, and the methods described involve the use of only simple apparatus.

There are at present four methods of artificial pacemaking in clinical use in the British Isles.

1. Long-term Electrode Catheter.-For this technique the patients carry a small pacemaker in a vest pocket or a neck sling, and this is attached to an electrode catheter which enters at the jugular vein and passes intravenously to the cavity of the right ventricle. The catheter may be "bipolar," with two electrodes at the tip, or
" unipolar," with a single-tip electrode. In the case of a unipolar catheter the second connexion from the pacemaker is made to an indifferent electrode buried in the subcutaneous tissue, usually in the neck.

2. Myocardial Wires with an External Unit.-In this case pacing is carried out through electrodes sutured surgically on to the heart and connected to a small external pacemaker by means of wires crossing the skin. This system is now rarely used for long-term pacing.

3. Completely Implanted Units.-In this technique a small selfcontained pacemaker is surgically implanted, usually in the rectus sheath, and is connected to the heart by wires which end in electrodes sutured on to the epicardium or into the cardiac muscle itself. The pacemaker contains mercury batteries with a life of two to five years, and at the end of this time the unit must be replaced surgically.

4. Induction Pacemaking.-A small receiving coil is implanted beneath the skin and connected to the heart by two electrode wires. The patient carries a small pacemaker connected to a similar coil, which is placed on the skin over the implanted coil. Power from the pacemaker is then transmitted to the heart by induction across the intact skin (Abrams, Hudson, and Lightwood, 1960).

* From the Cardiac Department, St. George's Hospital, London.

† Present address, Thorax Klin. (Fys.), Karolinska Sjukhuset, Stockholm, Sweden. 
We have only limited experience of the induction method of artificial pacemaking, and so this paper is confined to a discussion of the complications arising from the other three methods.

The patients in this series had been treated with a wide variety of different pacemakers, and eventually all the units used were designed by one of us (Davies, 1962). The techniques and threshold values described in this paper are applicable to patients using all makes of artificial pacemaker currently available in the British Isles.

All patients with artificial pacemakers should check their own pulse daily, and if there is any irregularity or alteration of rate by more than 3 beats $/ \mathrm{min}$. from the steady value they should contact their doctor for advice. The fault may be obvious-that is, broken wire externally-but if this is not the case the patient should be referred for an E.C.G. and a chest $x$-ray examination.

\section{Techniques}

E.C.G.-Two types of E.C.G. record are required. (1) A long strip of any lead is needed for detection of missed beats. This can be recorded on a direct-writing machine, but the stylus should be hotter than normal in order that the very rapid deflections due to the pacemaker stimulus are well shown. (2) Short strips of leads I and III are used for checking the polarity of pacemaker connexions. They should be recorded myocardial wires in place of the original unit. The voltage is then gradually reduced until pacing ceases and the threshold is read from the calibrated dial of the pacemaker; the threshold point can be determined by feeling the pulse, by a pulse monitor, or by a small oscilloscopic E.C.G. If a calibrated triggered oscilloscope is available threshold values can be determined more accurately, and, in addition, the voltage developed by the original pacemaker under working conditions can be measured.

It may be necessary for the pacemaker to be surgically exposed in order that the thresholds and the unit itself can be checked, and in this case a new sterile pacemaker should be available for replacement; when the urgency is too great for this to be done an attempt should be made to maintain absolute sterility of the myocardial wires and pacemaker site so that a new unit can be implanted later.

The threshold on each electrode of a bipolar system should be checked independently with the aid of a (positive) indifferent electrode, which may be a stainless-steel suture in the subcutaneous tissue, a metal retractor in the wound, or even an ordinary E.C.G. contact plate on the skin.

\section{Long-term Electrode Catheter}

For catheter pacing we use No. 5 solid C50 (unipolar) or C51 (bipolar) electrode catheters. ${ }^{1}$ Both types have a platinum

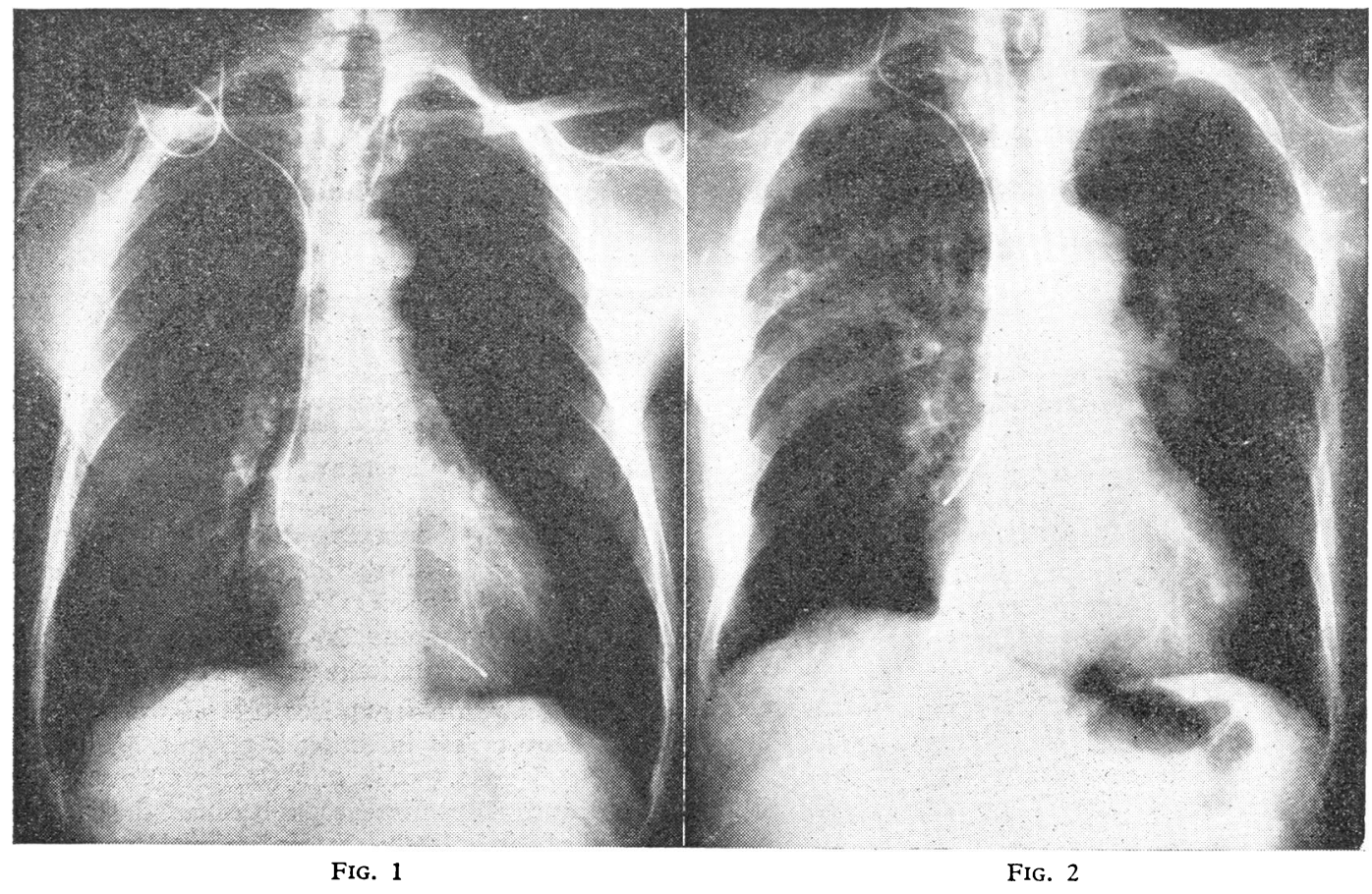

Fig. 1.-Unipolar electrode catheter in satisfactory position for long-term pacing: the tip is in apex of right ventricle and there are no loops. Frg. 2.-Electrode catheter tip displaced to right atrium.

photographically and should show the entire pacemaker stimulus: this may necessitate a sensitivity of $1 \mathrm{mv} . / \mathrm{mm}$. Maximum information from the E.C.G. records can be obtained if they can be compared with records taken previously during satisfactory pacing.

Chest X-ray Film.-The $x$-ray film should be "overpenetrated" to show the entire length of an electrode catheter or details of the structure of myocardial wires.

\section{Thresholds}

Simple threshold measurements may be made with the aid of a variable-voltage pacemaker which is connected to the contact at the tip, and the $\mathrm{C} 51$ has in addition a platinum ring contact $1 \mathrm{~cm}$. back from the tip. The catheter is passed from a jugular vein so that the contacts lie in the right ventricle, and in our experience the most suitable position is with the tip electrode impacted among the trabeculae near the apex (Fig. 1). Pacing via a bipolar catheter is often satisfactory even when the electrodes do not touch the endocardium, but it is unacceptable for the catheter to be looped or for the tip to lie in the pulmonary artery, the right atrium, the coronary sinus, or at the tricuspid valve. The electrode tip of a unipolar catheter must touch the endocardium for stable pacing. ${ }^{1}$ Made by the United States Catheter Corporation, Glenns Falls, New
York. 
Malposition of the electrode catheter is the most likely cause of unstable pacing in this group, although a recent study (Sowton, 1964) showed that repositioning of long-term electrode catheters was necessary only once in every 18 patientmonths. When this fault occurs the E.C.G. shows normal deflections due to the pacemaker stimuli, but these are followed only occasionally by a ventricular response.

Threshold measurements vary from moment to moment, and there is no clear end-point. The diagnosis is confirmed by chest $x$-ray examination, and the remedy lies in repositioning the catheter under $x$-ray control.

A rare but puzzling variation of the fault occurs when the catheter tip is in the right atrium (Fig. 2) in a patient in whom A-V conduction has returned. Pacing is often stable at rest, but the E.C.G. shows a delay between the pacemaker stimulus and the resulting QRS complex; if the pacemaker is turned off the underlying sinus rhythm is revealed (Fig. 3).

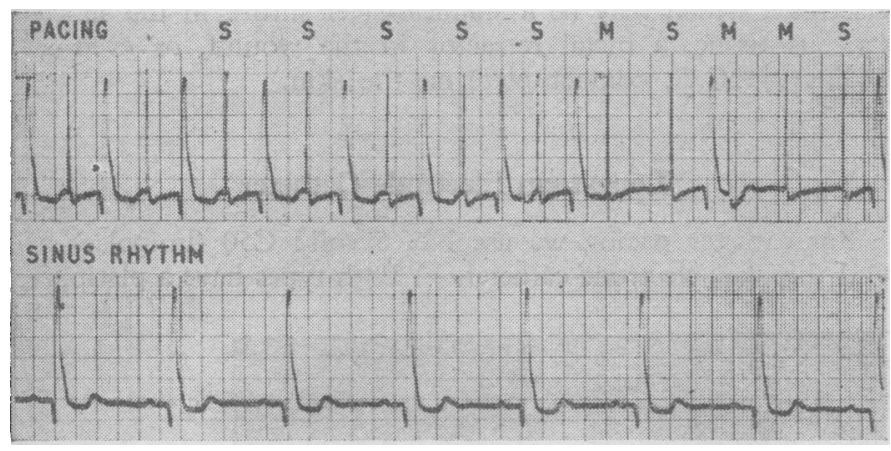

FIG. 3.-E.C.G.s taken on same occasion as $x$-ray film shown in Fig. 2. Lead II : Upper record : a delay of $0.3 \mathrm{sec}$. Occurs between the pacemaker stimulus and the corresponding QRS complex. $S=$ stimulus followed by ventricular response. $M=$ stimulus with no response (missed beat).
Lower record: sinus rhythm is confirmed when pacemaker is switched Lower record: sinus rhythm is confirmed when
off. $P-R$ interval 0.22 sec.

Broken wires or faulty connexions may be obvious on examination of the external wiring or may be hidden inside the catheter. The E.C.G. usually shows a very small or absent stimulus deflection, irrespective of which lead is recorded, and there is no ventricular response to the stimuli ; if the fault is intermittent there are occasional normal stimuli followed by paced beats. We have found that an internal fault in the wiring to the electrodes of a bipolar catheter is common, and this can be detected by the use of each electrode in turn for unipolar stimulation, with the aid of an indifferent electrode. If each catheter electrode appears satisfactory when used for unipolar pacing but bipolar pacing with both electrodes is impossible, then there is a short-circuit inside the catheter. A broken connexion inside a bipolar catheter almost invariably occurs in the wiring to the ring electrode, and so unipolar pacing can be continued via the tip electrode, provided the catheter position is satisfactory.

Perforation of the heart by an electrode catheter of the type we use is very rare, although it is a problem when stiffer catheters are employed. If this complication occurs, pacing becomes intermittent and may cease, while the E.C.G. shows a normal stimulus deflection with frequent failure of ventricular response. Chest pain is usually minimal or absent. The diagnosis is made from a chest $x$-ray film and the catheter should be repositioned under $x$-ray control. The patient should be closely observed for 24 hours after this has been carried out in case tamponade develops.

Infection around the entry site of the catheter is surprisingly rare, and usually responds to local measures. Septicaemia occurred three times in 120 catheterizations in this series, and was treated with antibiotics and removal of the catheter. On the whole, infection has proved much less of a problem than anticipated, and antibiotic cover is used only for the insertion and any subsequent manipulation of the catheter.

\section{Implanted Units}

Pacing by this means is the method of choice for most patients, but as the pacemaker and wiring are inaccessible investigation of faults is more difficult than with the two previous methods. A simple bedside test utilizing a portable radio can be applied to patients treated with most units available at present, and gives useful preliminary information. The radio is turned to maximum volume but is detuned between stations (optimal wavelength about 400 metres in the case of the pacemakers used in our patients) and is then applied closely over the site of the unit. A "clicking" from the loudspeaker indicates the discharge rate of the pacemaker, and can be compared with the pulse to detect missed beats. The only situation in which a discrepancy between the pacemaker rate and the ventricular rate is acceptable is in the presence of $A-V$ conduction, and this is considered in more detail below. The confirmation of sinus rhythm or the diagnosis of one of the following faults depends to a large extent upon the E.C.G.

Broken wires were very common with early electrodes of all types (Albert et al., 1962 ; Siddons, 1963 ; Chardack et al., 1963), but this fault has now become rare with the development of new materials and methods of production. It is sometimes possible to identify a break in internal wiring on a chest $x$-ray film (Parsonnet et al., 1963), but in general the site of the fault cannot be found by this means.

The E.C.G. picture depends upon the state of the insulation around the wire at the site of the break; if the insulation is intact the E.C.G. shows a small or absent stimulus deflection without ventricular response, but if the insulation is faulty the stimulus deflection may be normal, although there will be no resultant QRS complex. Pressure along the course of the wire may temporarily restore pacing by bringing the broken ends of wire into contact. The diagnosis is confirmed by threshold measurements taken after surgical exposure of the unit, and the remedy is for a spare myocardial wire to be utilized.

Infection or excess foreign-body reaction around an implantable unit usually results in discomfort post-operatively, which slowly increases over a few weeks or months until a sinus appears, with consequent relief of pain. Either before or after this occurs there is interference with pacing due to infection tracking up to the heart, although generalized pericarditis follows very rarely, if at all. Pacing is usually intermittent for some time before complete failure, and if the myocardial wires are exteriorized during this period a high threshold is found. This may fall considerably with free drainage and antibiotic treatment, but in our experience such measures merely prolong the time before long-term catheter pacing is necessary.

Rise in threshold requirements above the capabilities of the unit is rare unless infection is present. The normal threshold is below 3 volts (pulse width 2 msec.) over periods up to two years, and if pacing stops because the threshold has risen excessively it is likely that infection is present or that there is some fault in the connexions. In a few cases no fault can be found and pacing must then be continued with either a higheroutput pacemaker or via an electrode catheter.

The clinical and E.C.G. picture due to a very high threshold corresponds exactly with that due to loss of contact between the myocardium and the electrode; pacing ceases and the E.C.G. shows full-sized pacemaker stimuli occurring at the original rate, without any ventricular response.

Electrolytic destruction of stainless-steel electrodes occurs particularly, but not exclusively, with a faulty pacemaker and affects only the positive electrode. As this is often placed in the abdominal wall, this complication may present as tenderness over the site of the indifferent (positive) electrode ; the situation is usually complicated by sepsis, and the possibility of electrolysis should always be considered when an abscess is found confined to the region of the positive electrodes. Destruction of the electrodes causes loss of pacing, with almost complete disappearance of the stimulus deflection from the E.C.G. The 
remedy is to replace the pacemaker and positive electrodes, which should preferably be made of platinum.

Incorrect Pacemaker Polarity.-If a pacemaker is connected with the positive electrode on the heart and the negative as an indifferent electrode, pacing will be satisfactory initially but may later become erratic or cease. The E.C.G. can be used to determine the pacemaker polarity, and Fig. 4 shows leads I and III when the polarity is correct-that is, with the negative electrode on the heart and the positive in the abdominal wall-and shows that the initial deflection due to the pacemaker stimulus is negative in lead I and positive in lead III. This test can be carried out only on oscilloscopic or photographically recorded E.C.G.s and should be applied to every patient who has had an internal pacemaker with unipolar connexions implanted.

Alteration in pacemaker rate may occur because of battery exhaustion or because of a component fault. If the rate increases a dangerous tachycardia may result (Sowton, 1964 ; Parsonnet et al., 1963), and any pacemaker in an adult discharging at a rate of 115 beats $/ \mathrm{min}$. or over must be replaced at once.

\section{Return of A-V Conduction}

In $23 \%$ of our patients $\mathrm{A}-\mathrm{V}$ conduction returned soon after the onset of artificial pacing, with the production of parasystole. This situation should be suspected clinically when the pulse is of normal rate but irregular; the diagnosis is supported by an increasing pulse rate on exercise, and is finally confirmed by an E.C.G. There is an increased death rate among paced patients with parasystole which is almost certainly due to pace- maker-induced ventricular fibrillation (Sowton, 1964), but if pacing is stopped most of these patients revert to complete heart-block within four weeks. When pacing is to be continued for a patient in sinus rhythm the pacemaker rate should be slow (45-50 beats/min.), sympathomimetic drugs should be stopped as soon as pacing is established, and procainamide $250 \mathrm{mg}$. q.d.s. should be given for at least three days post-

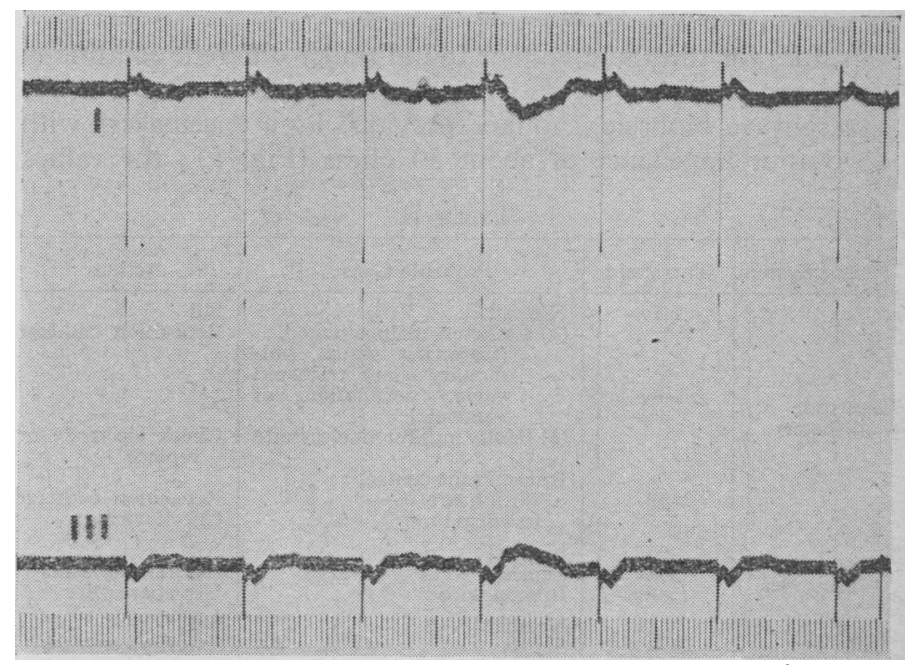

FIG. 4.-E.C.G. to check polarity of unipolar pacemaker connexions. Simultaneous leads I and III. The initial deflection due to pacemaker stimulus is negative in lead I and positive in lead III; this confirms that the negative electrode is in the heart and the positive electrode in the abdominal wall.

TABLE I.-Investigation of Pacemaker Failure

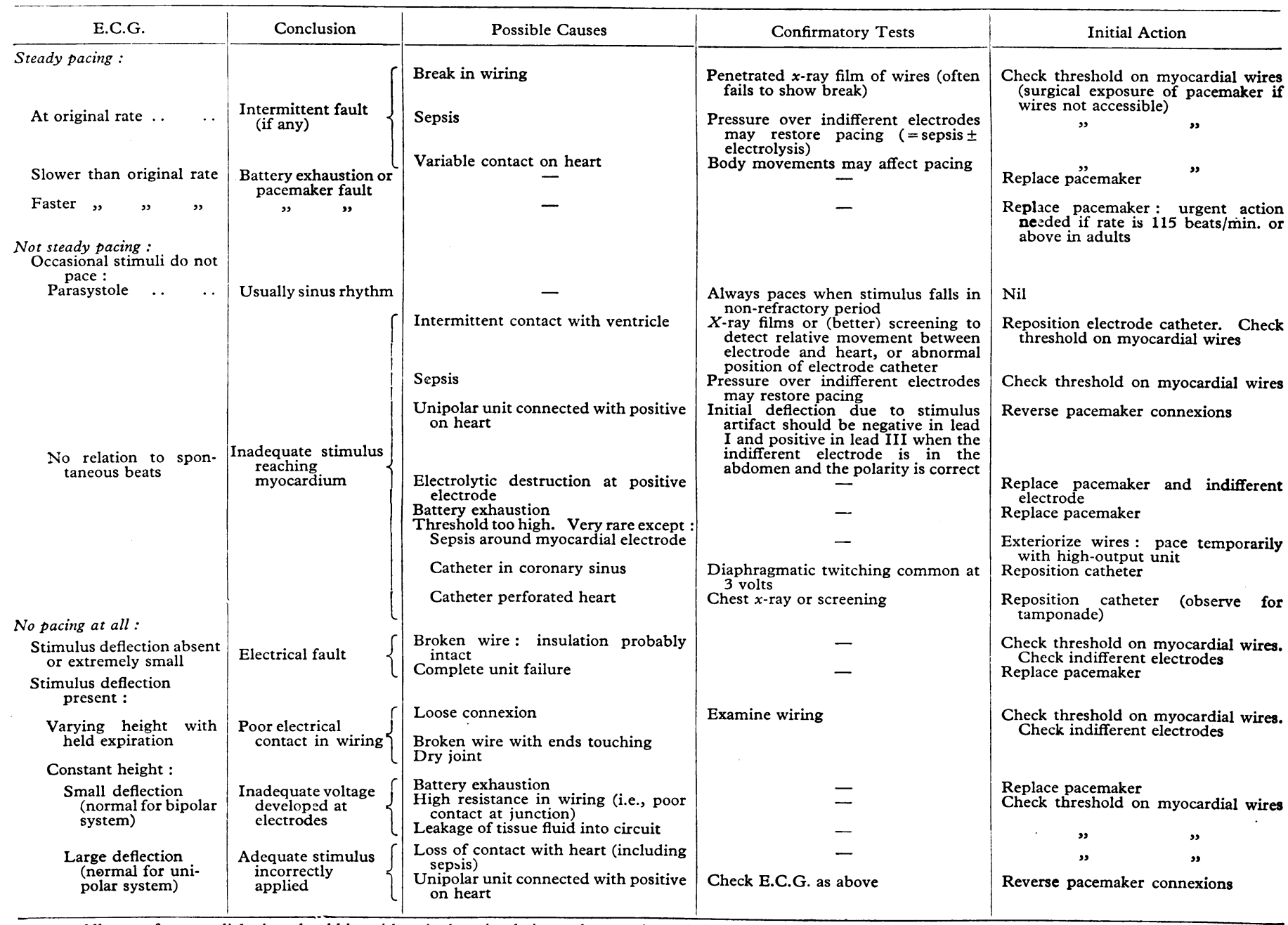


operatively ; new types of unit are at present being developed to meet the problem of competition between the natural and artificial pacemakers.

The analysis of E.C.G.s taken during the investigation of pacemaker failure can be carried out by reference to Table I. The importance of the deflection due to the pacemaker stimulus will be apparent, and it should be noted that the amplitude of this deflection is considerably less for bipolar stimulation than for unipolar stimulation.

Values for threshold voltage found under different conditions are given in Table II, and these were obtained with $2 \mathrm{msec}$. square-wave biphasic impulses provided by a pacemaker ${ }^{2}$ with an output impedance of about 50 ohms (Fig. 5); the values

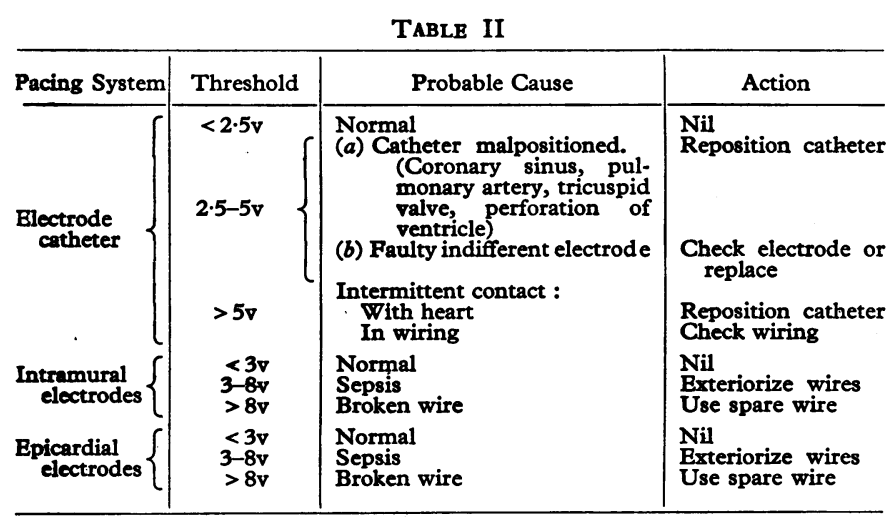

If no spare myocardial wire is available it may be necessary to abandon a bipolar system in favour of a unipolar system or to consider attachment of new myocardial wires to the heart; the long-term use of an electrode catheter may be preferable to a second thoracotomy in some patients.

with different equipment may be slightly higher. These figures refer to the thresholds after the electrode has been in position for three weeks or more; differences in threshold between bipolar and unipolar stimulation may be ignored for present purposes.

The work on which these readings are based will be fully reported elsewhere.

\section{Summary}

This paper provides a scheme for the initial investigation of patients treated with artificial pacemakers who present with a history suggestive of failure of pacing. The techniques of

${ }^{2}$ Obtainable from Devices Ltd., Welwyn Garden City, Herts, England. recording suitable E.C.G.s and $x$-ray films are described and the method of determining the approximate cardiac threshold with the aid of a simple adjustable pacemaker is reported. A rapid screening test of pacemaker function utilizing a portable radio is described. The interpretation of the results obtained is discussed in detail, with particular reference to some of the more common faults, including disturbance of pacing due to broken wires, battery failure, local infection, movement of electrode catheter position, and the return of $\mathrm{A}-\mathrm{V}$ conduction.

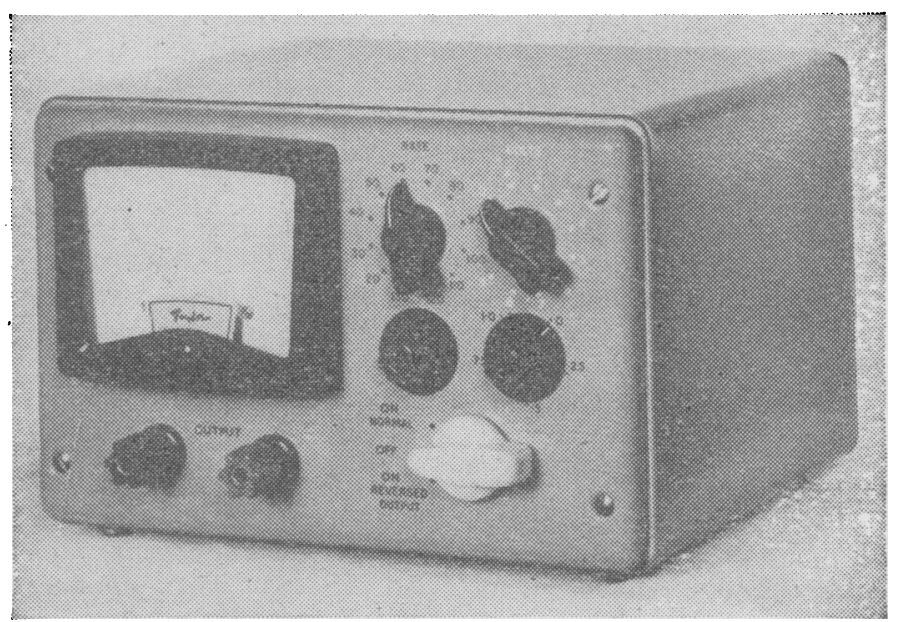

FIG. 5.-Pacemaker used for simple threshold determination. The voltage calibration remains accurate over long periods and the fine control is graduated in $\frac{1}{4}$-volt steps.

Our thanks are due to Dr. Aubrey Leatham and to Mr. A. H. M. Siddons for their encouragement: all the patients were admitted under their care.

REFERENCES

Abrams, L. D., Hudson, W. A., and Lightwood, R. (1960). Lancet, 1, 1372.

Albert, H. M., Glass, B. A., Andonie, J. A., and Cranor, K. C. (1962) Circulat. Res., 10, 295.

Chardack, W. M., Gage, A. A., Schimert, G., Thomson, N. B., Sanford, C. E., and Greatbatch, W. (1963). Dis. Chest, 43, 225 .

Davies, J. G. (1962). F. Brit. Instn Radio Engrs, 24, 6, 453.

Parsonnet, V., Gilbert, L., Zucker, R., and Asa, M. M. (1963). I. thorac. cardiovasc. Surg., 45, 801.

Siddons, A. H. M. (1963). Ann. roy. Coll. Surg. Engl., 32, 22.

Sowton, G. E. (1964). "Artificial Cardiac Pacemaking," M.D. Thesis, University of Cambridge.
* Medical Officer of Health, Harlow Urban District Council.

t Consultant Physician in Infectious Diseases, St. Ann's Hospital, Tottenham, and Rush Green Hospital, Romford.

₹ Conoultant Pathologist, St. Margaret's Hospital, Epping.

f Chief Public Health Inspector, Harlow Urban District Council.

\section{The Outbreak}

The infection came to light on 1 June 1963, when, as a result of laboratory tests, typhoid fever was diagnosed in four patients admitted to St. Margaret's Hospital, Epping, because of P.U.O. over the preceding two weeks. All general practitioners in the town were immediately alerted and asked to treat with suspicion every unexplained pyrexia. This resulted in more cases being suspected and confirmed during the ensuing days. In all, 26 persons were affected, but three of them had no clinical symptoms. Twenty-one came from eight households with two or more cases in each, and five were single cases. Their age and sex distribution is shown in Table I. 